Prepared for the U.S. Department of Homeland Security

Domestic Nuclear Detection Office

under U.S. Department of Energy Contract DE-AC05-76RL01830

\title{
PVT Degradation Studies: NMR Analysis
}

Revision 0

Herman Cho

Richard Kouzes

June 2017

Pacific Northwest

NATIONAL LABORATORY

Proudly Operated by Battelle Since 1965 


\title{
DISCLAIMER
}

This report was prepared as an account of work sponsored by an agency of the United States Government. Neither the United States Government nor any agency thereof, nor Battelle Memorial Institute, nor any of their employees, makes any warranty, express or implied, or assumes any legal liability or responsibility for the accuracy, completeness, or usefulness of any information, apparatus, product, or process disclosed, or represents that its use would not infringe privately owned rights. Reference herein to any specific commercial product, process, or service by trade name, trademark, manufacturer, or otherwise does not necessarily constitute or imply its endorsement, recommendation, or favoring by the United States Government or any agency thereof, or Battelle Memorial Institute. The views and opinions of authors expressed herein do not necessarily state or reflect those of the United States Government or any agency thereof.

\author{
PACIFIC NORTHWEST NATIONAL LABORATORY \\ operated by \\ BATTELLE \\ for the \\ UNITED STATES DEPARTMENT OF ENERGY \\ under Contract DE-AC05-76RL01830
}

Printed in the United States of America
Available to DOE and DOE contractors from the Office of Scientific and Technical Information,
P.O. Box 62, Oak Ridge, TN 37831-0062;
ph: (865) 576-8401
fax: $(865)$ 576-5728
email: reports@adonis.osti.gov

\begin{abstract}
Available to the public from the National Technical Information Service, U.S. Department of Commerce, 5285 Port Royal Rd., Springfield, VA 22161 ph: (800) 553-6847 fax: $(703) 605-6900$ email: orders@ntis.fedworld.gov online ordering: http://www.ntis.gov/ordering.htm
\end{abstract}

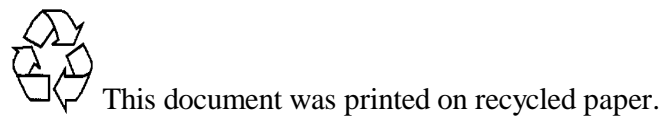




\title{
PVT Degradation Studies: NMR Analysis
}

\author{
Herman Cho \\ Richard Kouzes
}

June 2017

\author{
Prepared for \\ the U.S. Department of Homeland Security \\ Domestic Nuclear Detection Office \\ under U.S. Department of Energy Contract DE-AC05-76RL01830
}

Pacific Northwest National Laboratory

Richland, Washington 99352 


\section{Executive Summary}

Under certain environmental conditions, polyvinyl toluene (PVT) plastic scintillator is subject to internal fogging. Researchers have using nuclear magnetic resonance spectroscopy to elucidate the state of water inside the PVT.

The deuterium nuclear magnetic resonance results show that water absorbed by PVT under warm, humid conditions enters several distinct environments. When the PVT is transferred from incubation to ambient temperature and humidity, the water is lost on a time scale of a few hours from these samples. Most of the deuterium nuclear magnetic resonance peaks can be assigned to bulk liquid water. However, nearly $35 \%$ of the detected signal intensity is contained in a resonance that resembles spectra of water contained in nanometer-scale pores in mesoporous carbon.

This means that water likely occupies multiple sites within the PVT and its movement into or out of the bulk is governed by multiple time constants. 


\section{Contents}

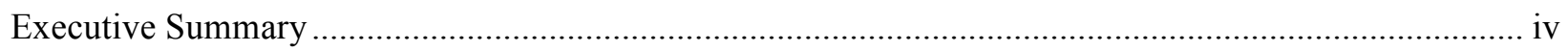

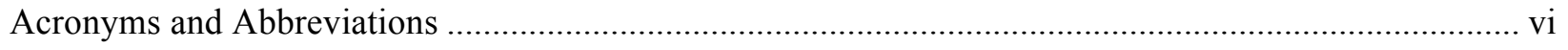

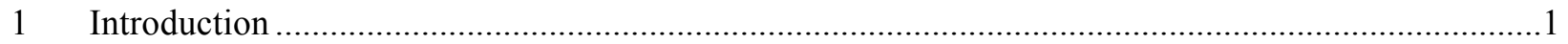

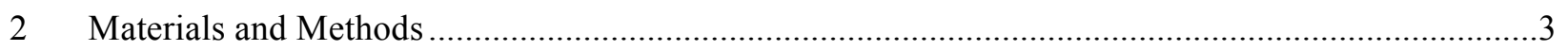

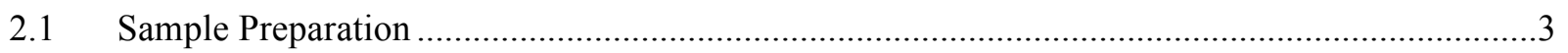

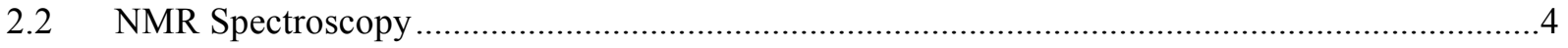

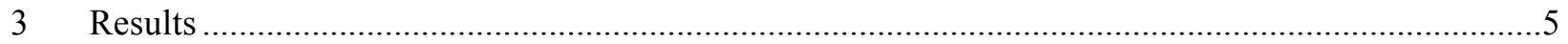

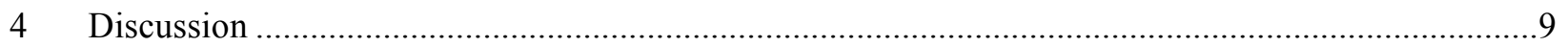

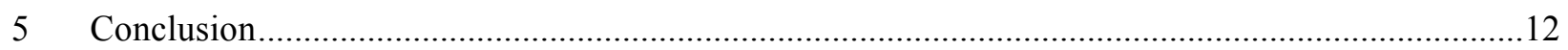

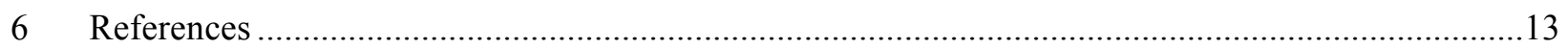

\section{Figures}

Figure 1-1: Inhomogeneously broadened ${ }^{2} \mathrm{H}$ NMR lineshape assuming an axially symmetric EFG tensor and isotropic (powder) orientational disorder. The frequency splitting parameter $V_{\mathrm{Q}}$ is equal to $3 e V_{\mathrm{zz}} Q / 2 h$, where $e$ is the fundamental charge, $V_{\mathrm{zz}}$ is the zz component of the EFG tensor, $Q$ is the quadrupolar moment of deuterium, and $h$ is Planck's constant.

Figure 2-1: Photograph of the disassembled magic angle spinning rotor used for ${ }^{2} \mathrm{H}$ NMR experiments and the pellet shaped PVT sample. The PTFE end cap inserts tightly and completely into the MAS sleeve, but is drilled through with an axial pinhole and does not hermetically seal the sample............ 3

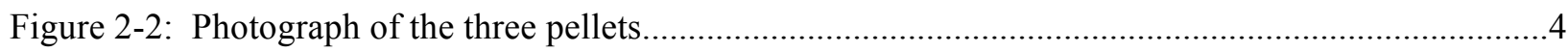

Figure 3-1: Deuterium NMR spectra of the $132 \mathrm{mg}$ PVT-A pellet recorded with single pulse excitation and at ambient temperature as a function of time after removal from ${ }^{2} \mathrm{H}_{2} \mathrm{O}$ incubation. The spectra have been deconvolved into four lines at -1.1, 4.3, 4.7, and $5.2 \mathrm{ppm}$, indicated by purple, blue, green, and black dashed lines, respectively......

Figure 3-2: Time evolution at room temperature of the integrated ${ }^{2} \mathrm{H}-\mathrm{NMR}$ intensities of the four lines fitted to the ${ }^{2} \mathrm{H}$ spectra of the PVT-A pellet (see Figure 3-1). Integrals of the -1.1, 4.3, 4.7, and 5.2 ppm deconvolved resonances are indicated by purple circles, blue squares, green triangles, and black crosses, respectively; solid lines with matching colors are least squares fits of the data to an exponential decay function. The summed intensities are shown as red triangles, and the sum of the line fits appears as a red solid line......

\section{Tables}

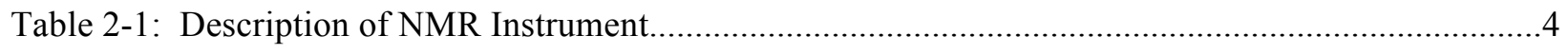

Table 3-1: Parameters from the fit of an exponential function to experimental data in Figure 3-2...........8 


\section{Acronyms and Abbreviations}

$\begin{array}{ll}{ }^{2} \mathrm{H} \text { or D } & \text { hydrogen-2 or deuterium } \\ \text { EFG } & \text { electric field gradient } \\ \text { MAS } & \text { magic angle spinning } \\ \text { NMR } & \text { nuclear magnetic resonance } \\ \text { PNNL } & \text { Pacific Northwest National Laboratory } \\ \text { PTFE } & \text { polytetrafluoroethylene (Teflon) } \\ \text { PVT } & \text { polyvinyl toluene (plastic scintillator) } \\ \text { RPL } & \text { Radiochemical Processing Laboratory }\end{array}$




\subsection{Introduction}

Polyvinyl toluene (PVT)-based gamma-ray detectors are widely used for many radiation detection applications. Studies show that over time, PVT exposed to weather conditions that span the range from extreme cold to extreme heat and a range of humidity degenerate in performance. This manifests itself as internal fogging of the PVT [Cameron 2015].

The water absorbed from environmental exposure (up to $0.06 \mathrm{wt} \%$ saturation at elevated temperatures) typically represents a small mass fraction of the hydrogen, oxygen, and water naturally present in organic solids. Therefore, experimental probes of such materials must be able to differentiate low quantities of absorbed, possibly reacted water, from a large intrinsic background. The use of rare hydrogen and oxygen isotopes (e.g. $\left.{ }^{2} \mathrm{H},{ }^{3} \mathrm{H},{ }^{17} \mathrm{O},{ }^{18} \mathrm{O}\right)$ provides a way to label and track absorbed water but requires a technique to selectively observe the rare isotope.

In the present study, we chose nuclear magnetic resonance (NMR) spectroscopy as our method to detect absorbed water enriched in the ${ }^{2} \mathrm{H}$ hydrogen isotope. Deuterium $\left({ }^{2} \mathrm{H}\right)$ is a stable isotope naturally present in concentrations of 156 atoms per million $\mathrm{H}$ atoms; however, water enriched in ${ }^{2} \mathrm{H}$ by more than a factor of $6400\left(99.9 \% \mathrm{D}_{2} \mathrm{O}\right)$ is readily available from commercial sources. The ${ }^{2} \mathrm{H}$ nuclide has magnetic properties $\left(I=1, \gamma=4106 \mathrm{rad} \mathrm{s}^{-1} \mathrm{G}^{-1}\right)$ that are favorable for NMR spectroscopy [Smith 1983]. In addition to being isotope specific, NMR experiments are rapid and non-destructive. They provide information on the quantities and chemical forms of deuterium. This can be valuable in investigations of the kinetics and reactions of water with PVT.

Deuterium is a spin-1 nuclide, which in an applied magnetic field has two allowed NMR dipole transitions: one between the +1 to 0 nuclear spin states, and the second between the 0 to -1 states. The energies of the two transitions due to the Zeeman interaction are degenerate. But they become unequal in the presence of a non-zero electric field gradient (EFG), which couples to the nuclear quadrupole moment to split the two transitions [Abragam 1961]. The resulting ${ }^{2} \mathrm{H}$ NMR spectrum has two lines separated by an energy proportional to the zz component of the EFG tensor. The so-called quadrupolar coupling is anisotropic. As a result, the magnitude of the splitting will depend on the orientation of the magnetic field direction with respect to the EFG tensor's principal axis system. For rigid, disordered solids, such as powders, the distribution of tensor orientations leads to an inhomogeneous broadening of the simple twoline spectrum of a spin-1 nuclide. The distinctive inhomogeneous lineshape for an ensemble of spin-1 nuclides with an isotropic distribution of fixed tensor orientations is known as a Pake pattern [Pake 1948]. This appears in Figure 1-1.

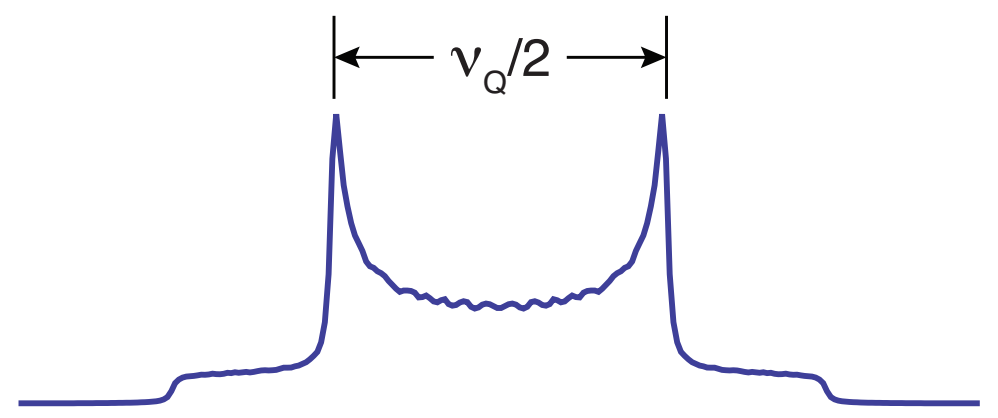

Figure 1-1: Inhomogeneously broadened ${ }^{2} \mathrm{H}$ NMR lineshape assuming an axially symmetric EFG tensor and isotropic (powder) orientational disorder. The frequency splitting parameter $V_{\mathrm{Q}}$ is equal to $3 e V_{\mathrm{zz}} \mathrm{Q} / 2 h$, where $e$ is the fundamental charge, $V_{\mathrm{zz}}$ is the zz component of the EFG tensor, $Q$ is the quadrupolar moment of deuterium, and $h$ is Planck's constant. 
For deuterium nuclei in rigid organic solids, the gradients of internal local fields produce splitting parameters $v_{Q}$ on the order of $10^{4}-10^{5} \mathrm{~Hz}$ [Smith 1983].

The NMR lineshapes of deuterium in dynamic environments deviate from the classic Pake pattern due to the modulation of the EFG tensor. In the presence of random reorientation of the nuclide, as occurs in Brownian motion in liquids, the time average of the EFG vanishes in the limit of short correlation times. Then the resonance in Figure 1-1 collapses to a single symmetric peak with a homogeneous lineshape described by the Lorentzian function

$$
I(v)=I_{0} \alpha^{2} /\left[\left(\alpha^{2}+\left(v-v_{0}\right)^{2}\right],\right.
$$

where $v_{0}$ is the center frequency of the line, $I_{0}$ is the height of the peak, and $2 \alpha$ is the full width of the peak at half-maximum [Abragam 1961], which for deuterium in aqueous solutions is typically of order $10^{2} \mathrm{~Hz}$ or less. The width and shape of deuterium NMR lines thus provide a way to determine the phase of ${ }^{2} \mathrm{H}$-bearing molecules, whether immobilized in a rigid solid or bound to a rapidly reorienting solution state molecule. 


\subsection{Materials and Methods}

\subsection{Sample Preparation}

Eljen Technology machined PVT samples in the form of pellets. These pellets (diameter $=3.43 \mathrm{~mm}$; length $=15 \mathrm{~mm}$ ) were made to fit magic angle spinning (MAS) NMR rotors (Pencil I design with $5 \mathrm{~mm}$ OD from Revolution NMR) (Figure 2-1). The sample is enclosed in the MAS sleeve with a polytetrafluoroethylene (PTFE) end cap. The use of pellet-shaped samples ensures a uniform mass distribution inside the rotor and smooth sample spinning. Pellet masses ranged from 130 to $132 \mathrm{mg}$.

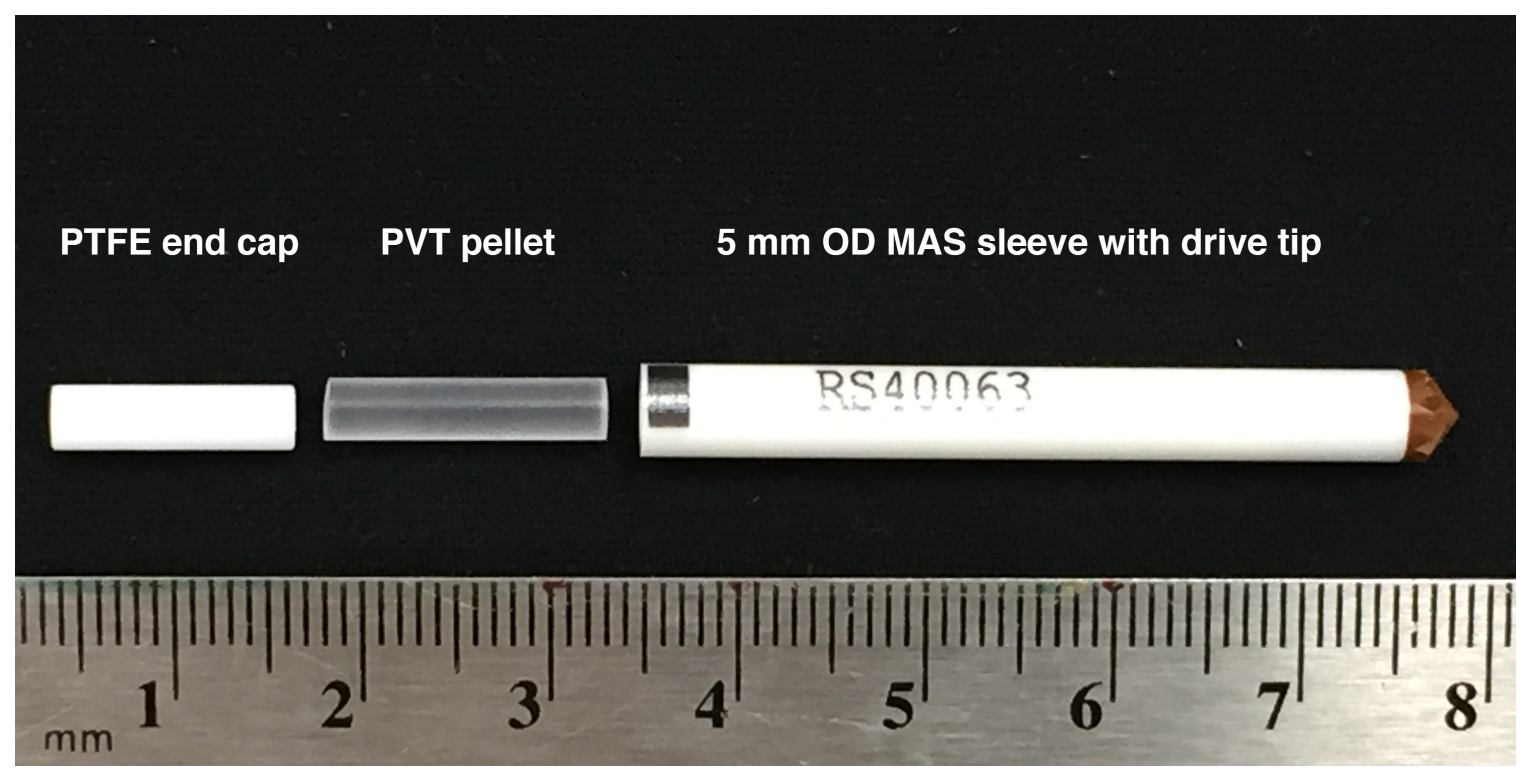

Figure 2-1: Photograph of the disassembled magic angle spinning rotor used for ${ }^{2} \mathrm{H}$ NMR experiments and the pellet shaped PVT sample. The PTFE end cap inserts tightly and completely into the MAS sleeve, but is drilled through with an axial pinhole and does not hermetically seal the sample.

We treated the as-received PVT pellets in three different ways to promote the incorporation of water (Figure 2-2). One pellet (PVT-A) was sealed in a vial partially immersed in $99.9 \%{ }^{2} \mathrm{H}$-enriched water (Cambridge Isotopes Laboratory, Inc.) and incubated at $65^{\circ} \mathrm{C}$ for 19 days. A second pellet (PVT-B) was prepared identically, but with non-enriched water; a third pellet (PVT-C) was incubated in an environmental chamber at a temperature of $65^{\circ} \mathrm{C}$ and saturation humidity conditions for 19 days. We observed no visible changes in water levels in the vials holding PVT-A and PVT-B during the incubation period.

Upon the end of the incubation period, the pellets were wiped dry and inserted in the NMR sample holder as quickly as possible. Pellets were handled with clean, dry tools to minimize surface contamination by water and other fluids. The ability of spectroscopic experiments to capture fast kinetic processes at $t=0$ is fundamentally determined by the interval between removal of the pellets from incubation to the start of the NMR signal acquisition. The entire process, which included loading and assembling the MAS rotor, transporting the sample to the NMR facility, and tuning the NMR instrument, typically required 15 minutes. 


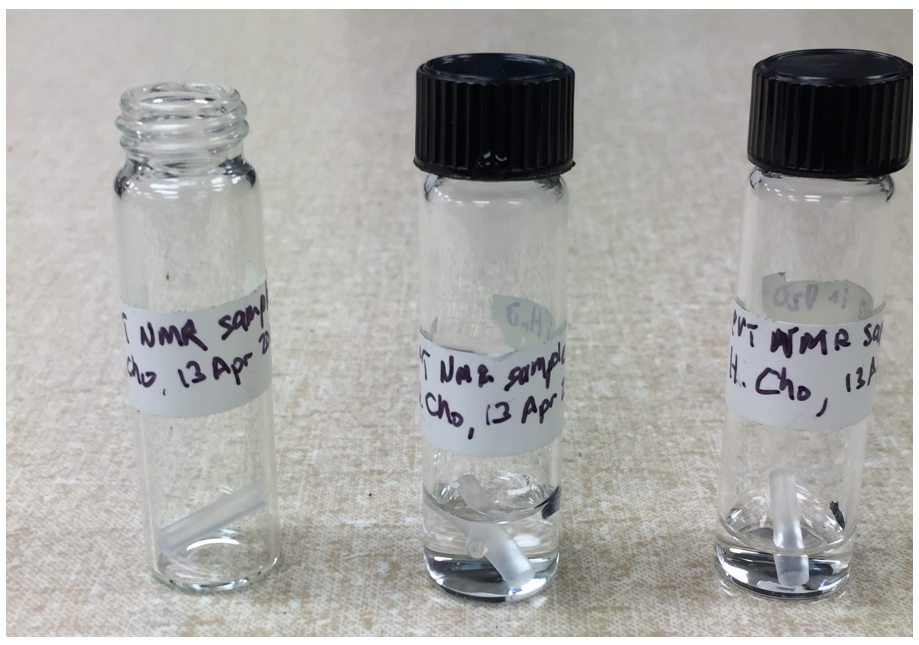

Figure 2-2: Photograph of the three pellets.

\subsection{NMR Spectroscopy}

We performed deuterium NMR experiments on the $300 \mathrm{MHz}$ spectrometer installed in the Radiochemical Processing Laboratory (RPL) at the Pacific Northwest National Laboratory. The main components of this instrument are listed in Table 2-1.

Table 2-1: Description of NMR instrument

\begin{tabular}{|l|l|}
\hline $\begin{array}{l}7.05 \text { Tesla superconducting widebore magnet } \\
\left(47.078 \mathrm{MHz}^{2} \mathrm{H} N M R \text { frequency) }\right.\end{array}$ & Oxford Instruments \\
\hline 34 channel room temperature shim set & Matrixshims, from Resonance Research Inc. \\
\hline Three channel broadband console & Redstone, from Tecmag, Inc. \\
\hline Probe & $5.0 \mathrm{~mm}$ HX MAS Pencil I, from Varian, Inc. \\
\hline
\end{tabular}

The chemical shift scale and the probe's $B_{1}$ field amplitude were calibrated with the ${ }^{2} \mathrm{H}$ signal of a $99.9 \%$ deuterium-enriched water sample. At $25^{\circ} \mathrm{C}$, the chemical shift of hydrogen isotopes in $\mathrm{H}_{2} \mathrm{O}$ is $4.766 \mathrm{ppm}$ [Wishart 1995], and the field amplitude was determined to be $78 \mathrm{kHz}\left(90^{\circ}\right.$ pulse width $\left.=3.2 \mu \mathrm{s}\right)$.

Deuterium NMR results presented in this report were acquired with single pulse excitation and scan recycle delays of $2 \mathrm{~s}$. Quadrupolar echo experiments with dephasing delays $\leq 300 \mu \mathrm{s}$, eight-step phase cycles, and scan delays up to $5 \mathrm{~s}$ were also performed in an effort to detect ${ }^{2} \mathrm{H}$ resonances with inhomogeneously broadened lineshapes characteristic of deuterium in solid phases.

In addition to the PVT pellets, the spectral region was scanned with rotor blanks and an empty probe to establish the background signal. We observed weak signals from deuterium in solid phases. We assign this to naturally occurring ${ }^{2} \mathrm{H}$ in the surrounding parts of the NMR probe made from organic materials.

The time evolution of the ${ }^{2} \mathrm{H}$ NMR spectrum of the PVT-A pellet was monitored by saving the averaged signal after 512 scans and restarting the signal averaging. A new spectrum was obtained every $1066 \mathrm{~s}$. This choice of experimental parameters represents a compromise between signal-to-noise ratio, which increases with high scan counts, and time resolution, which improves with low scan counts. 


\subsection{Results}

Deuterium NMR spectra of PVT-A appear in Figure 3-1. The resonances seen in this figure were absent from the pellets incubated with non-enriched water (PVT-B and PVT-C). We found no resonances displaying features characteristic of deuterium in a rigid solid. Spectra of PVT-A and PVT-C acquired with a quadrupolar excitation scheme were indistinguishable from blanks.

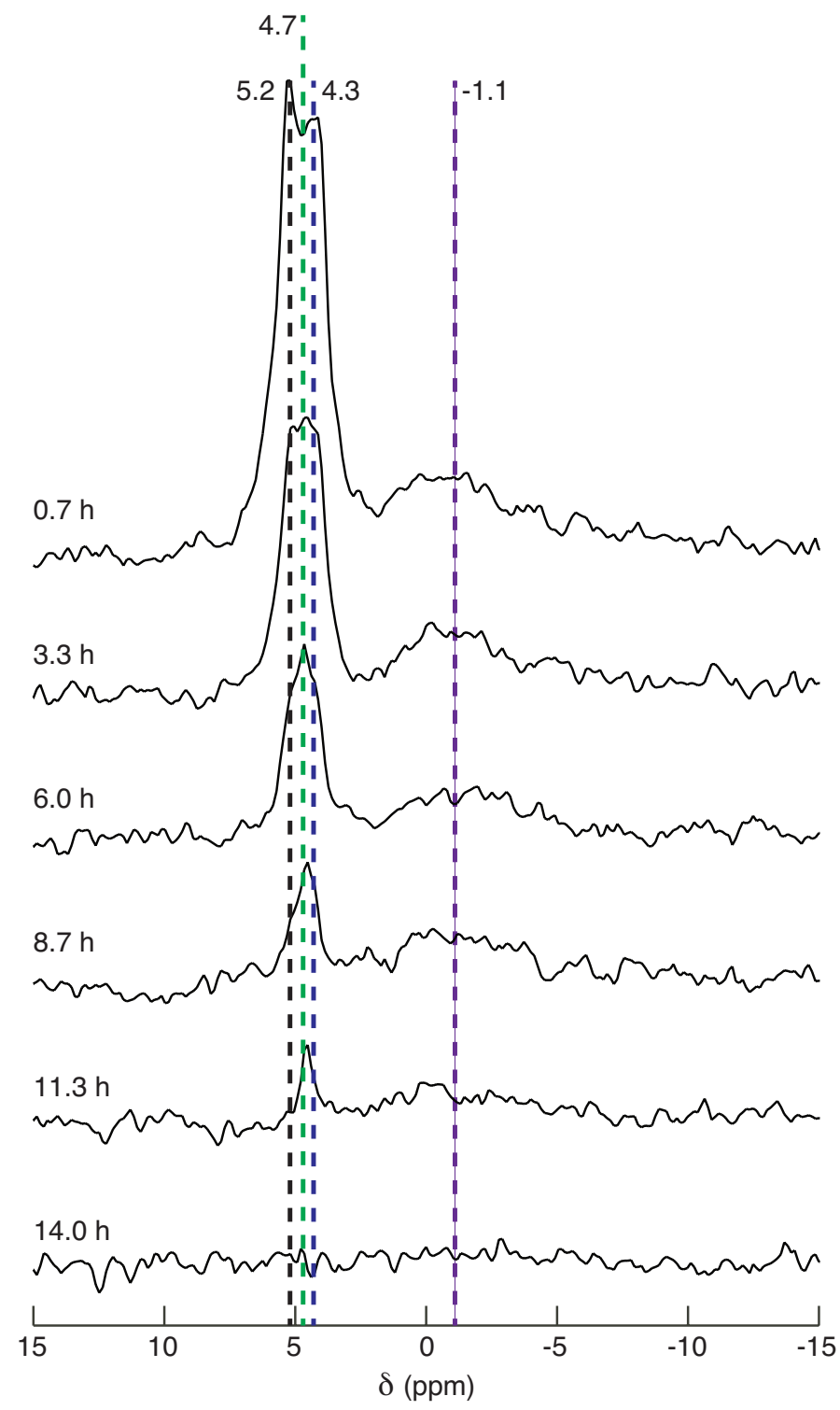

Figure 3-1: Deuterium NMR spectra of the $132 \mathrm{mg}$ PVT-A pellet recorded with single pulse excitation and at ambient temperature as a function of time after removal from ${ }^{2} \mathrm{H}_{2} \mathrm{O}$ incubation. The spectra have been deconvolved into four lines at $-1.1,4.3,4.7$, and $5.2 \mathrm{ppm}$, indicated by purple, blue, green, and black dashed lines, respectively.

The number of distinct resonances in these spectra is difficult to ascertain. This is particularly true in the band around 4.0 to $5.5 \mathrm{ppm}$, where several lines appear to overlap. A minimum of four Lorentzian lines (at $-1.1,4.3,4.7$, and $5.2 \mathrm{ppm}$ ) are required to obtain simulated spectra that fit the experimental results without systematic deviations in the residuals. According to Equation 1-2, each Lorentzian function is 
specified by three adjustable parameters: a chemical shift $\delta_{j}$; a relative amplitude; and a full width at half height linewidth.

As can be seen in Figure 3-1, the intensities of resolved spectral features decrease at different rates. The dependence of the integrated signal intensity on the time after removal from incubation is shown in Figure 3-2 for the four-line model.

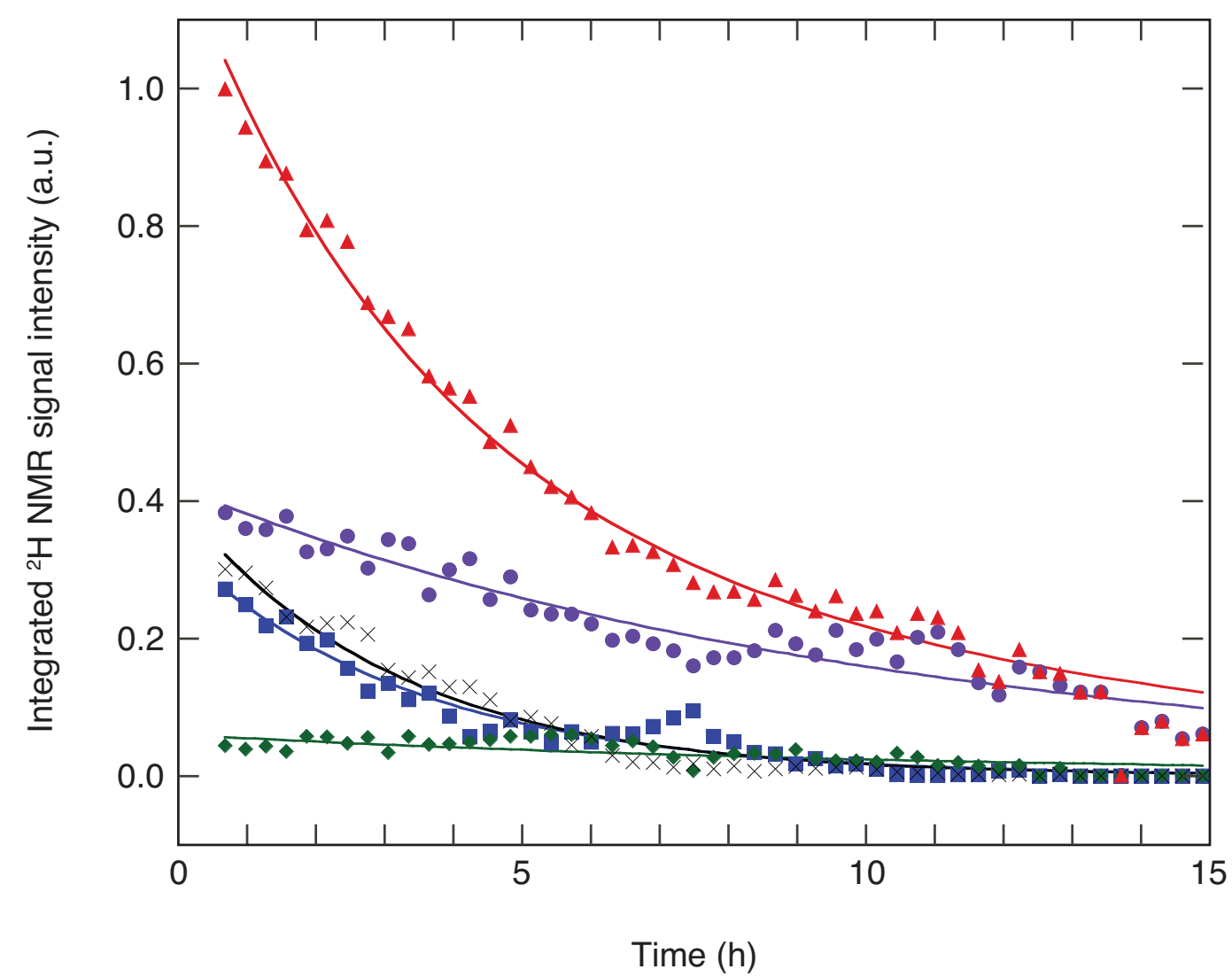

Figure 3-2: Time evolution at room temperature of the integrated ${ }^{2} \mathrm{H}-\mathrm{NMR}$ intensities of the four lines fitted to the ${ }^{2} \mathrm{H}$ spectra of the PVT-A pellet (see Figure 3-1). Integrals of the -1.1, 4.3, 4.7, and $5.2 \mathrm{ppm}$ deconvolved resonances are indicated by purple circles, blue squares, green triangles, and black crosses, respectively; solid lines with matching colors are least squares fits of the data to an exponential decay function. The summed intensities are shown as red triangles, and the sum of the line fits appears as a red solid line.

The intensity vs. time data of the four resonances were individually fitted to an exponential function of the form $I_{j}(t)=I_{j} \exp \left(-t / \tau_{j}\right)$, with adjustable parameters $I_{j}$ and $\tau_{j}$, and are plotted in Figure 3-2 with the experimental data. The parameters obtained from the least squares fits to the exponential function are compiled in 
Table 3-1. All of the time constants $\tau_{j}$ extracted from the fits exceeded the time increment of the kinetics measurement (1066 s or $0.3 \mathrm{~h})$ by a factor of ten or more. 
Table 3-1: Parameters from the fit of an exponential function to experimental data in Figure 3-2.

\begin{tabular}{|c|c|c|}
\hline $\begin{array}{c}\text { Line position } \boldsymbol{\delta}_{\boldsymbol{j}} \\
(\mathbf{p p m})\end{array}$ & $\begin{array}{c}\text { Exponential decay } \\
\text { constant } \boldsymbol{\tau}_{\boldsymbol{j}}(\mathbf{h})\end{array}$ & $\begin{array}{c}\text { Normalized } \\
\text { pre-exponential } \\
\text { coefficient } \boldsymbol{I}_{\boldsymbol{j}}\end{array}$ \\
\hline-1.1 & 10.30 & 0.347 \\
\hline 4.3 & 3.43 & 0.273 \\
\hline 4.7 & 10.75 & 0.050 \\
\hline 5.2 & 3.15 & 0.331 \\
\hline
\end{tabular}




\subsection{Discussion}

The only ${ }^{2} \mathrm{H}$ NMR peaks found in spectra of PVT-A (deuterium soaked) had widths and line shapes consistent with deuterium in a solution or gel phase. Although background deuterium in natural isotopic abundances would have been present in all three pellets, both in the solid and liquid states, the concentrations were below minimum detectable limits.

The presence of multiple discrete peaks indicates that absorbed water can enter several different environments in PVT. The resonances at 4.3, 4.7, and $5.2 \mathrm{ppm}$ are sufficiently close in their widths and chemical shifts to $\mathrm{D}_{2} \mathrm{O}(4.8 \mathrm{ppm})$ that an assignment to bulk water seems most plausible. The deviations from $4.8 \mathrm{ppm}$ can be attributed to interactions or reactions of water with PVT that result, e.g., in some water containing dissolved solids from the PVT that alter the chemical shift. The chemical shift of the peak at $4.3 \mathrm{ppm}$ fluctuated by almost $0.3 \mathrm{ppm}$ over the course of the kinetics experiment, while the peaks at 4.7 and 5.2 showed less variability.

The fourth line appears at a chemical shift (-1.1 ppm) that lies outside the known range for purely organic compounds. Hydrogen in organometallic molecules have been found with similar chemical shifts, but the requisite metals, including $\mathrm{Ga}, \mathrm{Se}, \mathrm{Li}$, are presumably not present in the PVT sample in sufficient concentrations. Xu et al. [2014] have observed ${ }^{1} \mathrm{H}$ NMR signals from mesoporous carbon materials that resemble the $-1.1 \mathrm{ppm}$ resonance in Figure 3-1, which they have assigned to water that enters small pores where they interact with aromatic molecules at the pore surface. The pore diameters in the mesoporous carbon solids studied by Xu et al. were between 3.57 and $3.85 \mathrm{~nm}$. The repeating structure of PVT contains a phenyl ring that according to the theory of $\mathrm{Xu}$ et al. could provide the magnetic shielding that shifts the ${ }^{2} \mathrm{H}$ resonance in the observed direction, i.e., to lower energy. Alternatively, reactions of water with nominally inert solids such as glasses have been found to form gels at the interfaces that exhibit broad NMR peaks at unusual chemical shifts.

The relative initial populations of deuterium nuclei in each environment can be estimated from the parameters $I_{j}$ in 
Table 3-1, which correspond to the normalized integrated intensity of each peak at $t=0$. No single peak predominates, although $I_{j}$ for the $4.7 \mathrm{ppm}$ resonance, which lies closest to the chemical shift of pure bulk water, is less than one-fifth as large as the other three.

The exponential decay constants $\tau_{j}$ in 
Table 3-1 indicate that the 4.3 and $5.2 \mathrm{ppm}$ peaks disappeared at a rate more than three times faster than rates for the -1.1 and $4.7 \mathrm{ppm}$ resonances. In the case of the $-1.1 \mathrm{ppm}$ peak, the slower rate is consistent with the hypothesis that water is weakly bound inside pores and must diffuse out of a confined volume prior to evaporation. More generally, the rate at which water leaves the PVT matrix will be influenced by an array of extrinsic factors, including temperature, the size and shape of the PVT monolith, the surface to volume ratio, encapsulation inside a container (e.g., the MAS rotor), etc. The absolute values of the decay constants should therefore not be considered fixed properties of the water in PVT; nevertheless, the relative magnitudes give one measure of how tightly the PVT matrix holds absorbed water in each of the possible environments. 


\subsection{Conclusions}

The conclusions of our work are as follows.

Water time constants. The deuterium NMR results presented above show that water absorbed by PVT (at least the sample tested) under warm, humid conditions enters several distinct environments. Furthermore, when the PVT is transferred from incubation to ambient temperature and humidity, the water is lost on a time scale of a few hours from these samples.

Nanometer-scale pores. Most of the deuterium NMR peaks can be assigned to bulk liquid water, but almost $35 \%$ of the detected signal intensity is contained in a resonance that resembles spectra of water confined to nanometer-scale pores in mesoporous carbon. Surface area measurements using the method of Barrett-Joyner-Halenda [1951] would be informative in determining the volume available, if any, in such pores. In order to evaluate the true capacity of pores for water uptake, samples in future experiments should be thoroughly dehydrated to extract residual water before incubation.

More work needed on deuterium quantification. The absolute amounts of deuterated water absorbed by PVT-A were not measured in the experiments discussed here. Such measurements require intensity standards with comparable concentrations of ${ }^{2} \mathrm{H}$ and some knowledge of the rate of water loss. This information was not known prior to this work, but the data contained in this report should prove to be a useful guide for designing future experiments for absolute deuterium quantification by NMR spectroscopy. The ability of NMR spectroscopy to quantify water in individual environments could also provide insights on the reversibility of hydration/dehydration cycles in PVT.

More data on relaxation time. Relaxation time measurements could resolve several questions on the nature of the bulk water and the trapping of water molecules in pores. However, the short time window available to perform experiments before the water has disappeared limits the experiments that can be performed. This also affects the signal-to-noise ratio improvement that can be achieved with scan averaging. 


\subsection{References}

[Abragam 1961] A Abragam, Principles of Nuclear Magnetism (Clarendon, Oxford, 1961).

[Barrett 1951] EP Barrett, LG Joyner, and PP Halenda, J. Am. Chem. Soc. 73, 373 (1951).

[Kunwar 1986] AC Kunwar, GL Turner, and E Oldfield, J. Magn. Reson. 69, 124 (1986).

[Pake 1948] GE Pake, J. Chem. Phys. 16, 327 (1948).

[Smith 1983] ICP Smith, in NMR of Newly Accessible Nuclei, Vol. 2, edited by P Laszlo (Academic Press, New York, 1983) pp. 1-26.

[Wishart 1995] DS Wishart, CG Bigam, J Yao, F Abildgaard, HJ Dyson, E Oldfield, JL Markley, and BD Sykes, J. Biomol. NMR 6, 135 (1995).

[Xu 2014] Y Xu, T Watermann, H-H Limbach, T Gutmann, D Sebastian, and G Buntkowsky, Phys. Chem. Chem. Phys. 16, 9327 (2014). 


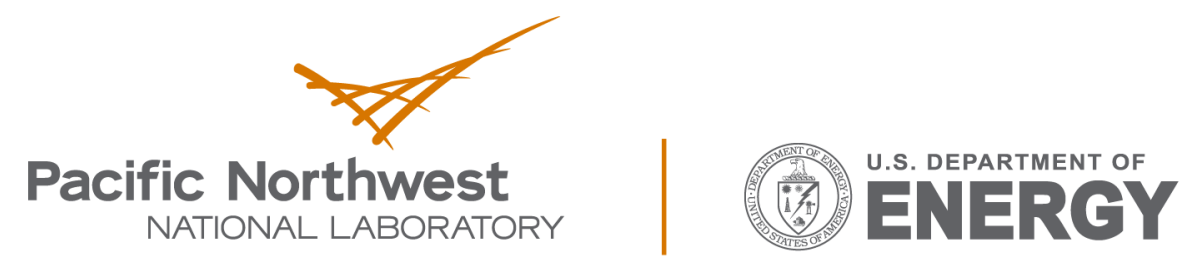

Proudly Operated by Battelle Since 1965

902 Battelle Boulevard

P.O. Box 999

Richland, WA 99352

1-888-375-PNNL (7665)

www.pnnl.gov 